\title{
Elastografía por ondas de corte como herramienta en la evaluación de los nódullos tiroideos
}

Carlos Paredes-Manjarrez, ${ }^{1}$ Demetrio Arreola-Cháidez, ${ }^{\text {* }}$ Andrés Magdalena-Buitrago, ${ }^{2}$

Aldo Ferreira-Hermosillo, ${ }^{1}$ José F. Avelar-Garnica ${ }^{1}$ y Rocío Arreola-Rosales ${ }^{1}$

${ }^{1}$ Instituto Mexicano del Seguro Social, Centro Médico Nacional Siglo XXI, Unidad de Investigación Médica en Enfermedades Endocrinas, Ciudad de México, México; ${ }^{2}$ Instituto Prestador de Salud, Diagnóstico e Imágenes del Valle, Cali, Colombia

\section{Resumen}

Introducción: La elastografía por ondas de corte (SWE) ha demostrado ser predictiva de malignidad en nódulos tiroideos. Objetivo: Determinar mediante SWE, el punto de corte de la rigidez con mayor especificidad y sensibilidad para detectar nódulos tiroideos que requieren cirugía. Métodos: Estudio transversal de pacientes con nódulos tiroideos evaluados ultrasonográficamente en un periodo de tres años; se empleó la clasificación TI-RADS y mediante SWE se determinó la rigidez de los nódulos. Con el sistema Bethesda se clasificaron las muestras histopatológicas y mediante curva ROC se obtuvo el punto de corte de la rigidez con mayor especificidad y sensibilidad. Resultados: $41 \%$ de los nódulos fue TI-RADS 5 y 59 \%, TI-RADS 1-4. En los TI-RADS 5, la mediana de rigidez de los nódulos con categoría IV-VI del sistema Bethesda fue de $35.9 \mathrm{kPa}$ y en los nódulos con TI-RADS 1-4, $21.6 \mathrm{kPa}$. En los nódulos TI-RADS 5, la rigidez > $32.5 \mathrm{kPa}$ tuvo especificidad de $75 \%$ y sensibilidad de $57 \%$ para detectar los que requieren cirugía; en los TI-RADS 1-4, el valor de corte de $21.5 \mathrm{kPa}$ tuvo especificidad de $63 \%$ y sensibilidad de $51 \%$. Conclusión: La rigidez determinada por SWE es útil para detectar nódulos que requerirán exploración quirúrgica.

PALABRAS CLAVE: Cáncer de tiroides. Ultrasonografía. Nódulo tiroideo.

\section{Shear-wave elastography as a tool in the assessment of thyroid nodules}

\section{Abstract}

Introduction: Shear-wave elastography (SWE) has been shown to be predictive of malignancy in thyroid nodules. Objective: To determine, by SWE, the stiffness cutoff point with the highest specificity and sensitivity to detect thyroid nodules that require surgery. Methods: Cross-sectional study of ultrasonographically-evaluated patients for thyroid nodules over a period of three years; the TI-RADS classification system was used, and nodule stiffness was determined by SWE. Histopathological specimens were classified using the Bethesda system, and the stiffness cutoff point with the highest specificity and sensitivity was obtained using ROC curves. Results: Forty-one percent of the nodules were classified as TI-RADS 5, and $59 \%$, as TI-RADS 1-4. In TI-RADS 5 nodules, median stiffness of those in Bethesda system IV-VI categories was $35.9 \mathrm{kPa}$; in nodules with TI-RADS 1-4, $21.6 \mathrm{kPa}$. In TI-RADS 5 nodules, a cutoff point > $32.5 \mathrm{kPa}$ had a specificity of $75 \%$ and sensitivity of $57 \%$ to detect those requiring surgery; in TI-RADS 1 to 4 nodules, a cutoff point of $21.5 \mathrm{kPa}$ had a specificity of $63 \%$ and sensitivity of $51 \%$. Conclusion: SWE-determined stiffness is useful to detect nodules that require surgical evaluation.

KEY WORDS: Thyroid cancer. Ultrasonography. Thyroid nodule.

Correspondencia:

*Demetrio Arreola-Cháidez

E-mail: demetrioii@ hotmail.com
Fecha de recepción: 28-11-2019

Fecha de aceptación: 26-05-2020

DOI: $10.24875 / G M M .20005656$
Gac Med Mex. 2021;157:19-24

Disponible en PubMed

www.gacetamedicademexico.com

0016-3813/@ 2020 Academia Nacional de Medicina de México, A.C. Publicado por Permanyer. Este es un artículo open access bajo la licencia CC BY-NC-ND (http://creativecommons.org/licenses/by-nc-nd/4.0/). 


\section{Introducción}

Los nódulos tiroideos son los tumores endocrinológicos más frecuentes, $95 \%$ es de naturaleza benigna y $5 \%$, neoplasias, principalmente cáncer papilar de tiroides. ${ }^{1}$ De acuerdo con la Organización Mundial de Salud, la prevalencia de cáncer tiroideo en el mundo es de 1977846 casos, con incidencia de 567233 casos. En México, representa el segundo cáncer con más prevalencia, con un total de 40770 casos en 2018. ${ }^{2}$

Para la estadificación del riesgo de malignidad, los nódulos tiroideos se clasifican conforme a la escala TI-RADS (Thyroid Imaging Reporting and Data System) de acuerdo con sus características ultrasonográficas. ${ }^{3}$ En 2017, Ha et al. analizaron los criterios TI-RADS publicados por la ACR (American College of Radiology), la ATA (American Thyroid Association ) y la KTA/KSThR (Korean Thyroid Association/Korean Society of Thyroid Radiology); demostraron que los criterios de la ACR tenían la especificidad más alta (75\%) para la detección de malignidad, con las tasas más bajas de biopsias innecesarias $(21 \%) .{ }^{4}$ En abril de 2017, la ACR realizó una actualización llamada ACR TI-RADS. ${ }^{5}$

La biopsia con aspiración con aguja fina (BAAF) es el estándar de oro para determinar si los nódulos tiroideos son benignos o malignos. El resultado es clasificado con el sistema Bethesda, el cual establece seis categorías en función del porcentaje del riesgo de malignidad: 6

- I, insatisfactorio.

- II, benigno (0 a $3 \%$ ).

- III, atipia de significado indeterminado (5 a $15 \%$ ).

- IV, neoplasia folicular (15 a $30 \%$ ).

- V, sospecha de malignidad (60 a $75 \%$ ).

- VI, malignidad (97 a $99 \%$ ).

Sin embargo, la BAAF es un método invasivo que puede presentar complicaciones locales o resultados falsos negativos hasta en $15 \%$ y que requiere la repetición del procedimiento si la muestra es insuficiente. ${ }^{7,8}$

La toma de biopsia guiada por elastografía ha ayudado a aumentar la capacidad para diferenciar entre nódulos tiroideos benignos y malignos. La elastografía por ultrasonido es un método de imagen que mide la elasticidad y rigidez de los tejidos ${ }^{9}$ y que en los últimos 20 años se ha propuesto para diferenciar las neoplasias malignas en diferentes tejidos, ${ }^{10}$ con la ventaja de ser poco costosa y no invasiva.
La elastografía cuantitativa es una técnica reciente disponible en sistemas de ultrasonido avanzado. Consiste en la obtención de imágenes con la medición del desplazamiento en los tejidos de las señales ultrasónicas pre y posaplicación de fuerza compresiva: a la compresión, los tejidos enfermos exhiben menor deformación que los tejidos sanos. ${ }^{11}$ Tanto la elastografía en tiempo real como por ondas de corte (SWE, shearwave elastography) han demostrado ser predictivas de malignidad en nódulos tiroideos..$^{12}$ En México, su papel definitivo aún se encuentra en evaluación.

\section{Métodos}

Se realizó un estudio de cohorte retroprolectivo en un periodo de tres años (enero de 2017 a julio de 2019) en los pacientes del Servicio de Ultrasonido del Hospital de Especialidades, Centro Médico Nacional Siglo XXI, Instituto Mexicano del Seguro Social, de uno y otro sexo mayores de 18 años, referidos para la realización de BAAF de nódulos tiroideos. Se excluyeron pacientes con diagnóstico previo de cáncer tiroideo, con estudio de imagen incompleto o con una muestra de BAAF inadecuada para el diagnóstico patológico (Bethesda I), si esta no podía ser repetida.

\section{Estudio ecográfico}

En todos los pacientes se realizó evaluación ecográfica de la glándula tiroides con ultrasonido en modo B en escala de grises y ecografía Doppler color para valorar composición, ecogenicidad, forma, márgenes y calcificaciones asociadas. Con dichas características, los nódulos fueron clasificados conforme el sistema TI-RADS: ${ }^{5}$

- Benigno, cero puntos.

- No sospechoso, dos puntos.

- Levemente sospechoso, tres puntos.

- Moderadamente sospechoso, cuatro a seis puntos.

- Altamente sospechoso, siete o más puntos.

Para la evaluación de la utilidad de la SWE se compararon los nódulos clasificados como TI-RADS 5 versus TI-RADS 1-4. La elastografía se realizó con equipo Aixplorer ${ }^{\circledR}$ (Supersonic Imagine, Aix-enProvence, Francia), con un transductor lineal que emplea una frecuencia de $18 \mathrm{MHz}$. Los valores de la elastografía se evaluaron con el programa Sonic Touch $^{\mathrm{TM}}$ Technology (Supersonic Imagine, Provence, 
Francia), que emplea cinco regiones de interés (cinco muestras de la rigidez nodular) de 2 a $5 \mathrm{~mm}$ de diámetro y reporta la media de los kilopascales $(\mathrm{kPa})$ medidos.

\section{BAAF de tiroides}

Previa asepsia y antisepsia se infiltró $1 \mathrm{~mL}$ de lidocaína a $2 \%$ en los tejidos blandos. Mediante guía ecográfica se realizó punción percutánea hasta alcanzar el nódulo y mediante aspiración con aguja calibre $20 \mathrm{G}$ se obtuvo material biológico, el cual se colocó en portaobjetos para realizar frotis y se fijó en alcohol absoluto; las muestras fueron evaluadas por patólogos del hospital que reportaron el resultado de la citología con el sistema Bethesda. ${ }^{6}$ En nuestra unidad, los nódulos tiroideos estratificados como II y III conforme la clasificación Bethesda requieren únicamente vigilancia, mientras que aquellos clasificados como IV, V y VI requieren exploración quirúrgica: IV y $\mathrm{V}$ requieren hemitiroidectomía y $\mathrm{VI}$, tiroidectomía total.

\section{Análisis estadístico}

Los datos se analizaron con el programa SPSS versión 23. Se valoró la normalidad de las variables con la prueba de Kolmogorov-Smirnov. Debido a que se trata de variables no paramétricas, los resultados cuantitativos fueron expresados con medianas y rangos intercuartílicos. Se utilizó ANOVA para evaluar las diferencias entre los grupos según su clasificación por TI-RADS. Para evaluar las diferencias entre los nódulos TI-RADS 1-4 versus los nódulos TI-RADS 5 se utilizó $U$ de Mann-Whitney. Las variables cualitativas se expresaron con porcentajes y las diferencias entre los grupos fueron evaluadas mediante chi cuadrada en caso de dos grupos y mediante prueba de McNemar en caso de tres o más grupos. Para determinar los puntos de corte de la SWE para la detección de nódulos que requieren abordaje quirúrgico (clasificados como IV, V o VI del sistema Bethesda) se realizaron curvas ROC (receiver operating characteristics). Los puntos de corte fueron seleccionados con el índice de Youden (sensibilidad + especificidad -1$){ }^{13}$

\section{Aspectos éticos}

El protocolo fue aprobado por el Comité Local de Investigación y Ética en Investigación en Salud. Los pacientes firmaron la carta de consentimiento informado para la realización de los procedimientos y para el análisis de su información, después de explicarles los objetivos del estudio. Los procedimientos respetaron las normas éticas establecidas en la Declaración de Helsinki.

\section{Resultados}

De 756 pacientes revisados por nódulo tiroideo, $41 \%(n=309)$ fueron clasificados por ecografía como TI-RADS 5 de la ACR y $59 \%(n=449)$, como TI-RADS 1-4; $90 \%$ de la población total fue mujer y la mediana de edad fue 57 años (46-66 años). En la Tabla 1 se observan las características demográficas y ecográficas de la población conforme su clasificación TI-RADS.

En cuanto a las variables ecográficas según la clasificación TI-RADS, no se observaron diferencias significativas en el diámetro mayor del nódulo; de hecho, los nódulos tiroideos TI-RADS 5 son más pequeños (mediana de $1.41 \mathrm{~cm}$ ) en comparación con los clasificados como TI-RADS 1 (mediana de $2.05 \mathrm{~cm}$ ). Se observó diferencia entre los márgenes de los nódulos dependiendo de la clasificación TI-RADS, ya que entre más baja era esta (1 y 2) se observaron bordes lisos, en contraste con los nódulos grados TI-RADS 4 y 5 , que presentaron márgenes lobulados y, algunos de ellos, extensión extratiroidea. Otra variable diferente entre los grupos fue la composición: el componente predominantemente quístico se observó en la totalidad de los nódulos TI-RADS 1 (100\%) y solamente en $1 \%$ de los TI-RADS 5; en tanto que el componente predominantemente sólido se encontró en $79 \%$ de los nódulos TI-RADS 5 y en ninguno de Ios TI-RADS 1 y 2.

El resultado citopatológico de los nódulos considerados como TI-RADS 1 y 2 fue en su mayoría benigno (categorías Bethesda II y III), mientras que el porcentaje de reportes Bethesda IV a VI se incrementó en los estadios TI-RADS 3 a $5(p<0.001)$. Se registró Bethesda I (muestra insuficiente) en $21 \%$ de los nódulos en los que se realizó biopsia, lo cual no fue estadísticamente diferente entre los grupos, independientemente de su clasificación TI-RADS (Tabla 1). Los pacientes con nódulos Bethesda I fueron eliminados de los análisis estadísticos posteriores. Al agrupar los nódulos por TI-RADS 5 y TI-RADS 1-4, así como por Bethesda I, II-III y IV-VI, aquellos clasificados como TIRADS 5 se distribuyeron de la siguiente manera: $20 \%$ se clasificó como Bethesda I, 38 \% como Bethesda II-III y $42 \%$ como Bethesda IV-VI. En cuanto a los nódulos TIRADS 1-4, la distribución fue de la 
Tabla 1. Características demográficas y ecográficas de la población

\begin{tabular}{|c|c|c|c|c|c|c|}
\hline Variables & $\begin{array}{l}\text { TI-RADS } 1 \\
(\mathrm{n}=30)\end{array}$ & $\begin{array}{c}\text { Tl-RADS } 2 \\
(n=58)\end{array}$ & $\begin{array}{l}\text { TI-RADS } 3 \\
(n=101)\end{array}$ & $\begin{array}{l}\text { TI-RADS } 4 \\
(n=260)\end{array}$ & $\begin{array}{l}\text { TI-RADS } 5 \\
(n=309)\end{array}$ & $p$ \\
\hline Edad en años ${ }^{\ddagger}$ & $64(55-68)$ & $52(44-65)$ & $53(42-65)$ & $59(46-68)$ & $57(45-64)$ & NS \\
\hline Sexo (\% mujeres) & 90 & 97 & 94 & 84 & 87 & NS \\
\hline $\begin{array}{l}\text { Diámetro mayor de la } \\
\text { lesión }(\mathrm{cm})^{\ddagger}\end{array}$ & $2.05(0.80-2.53)$ & $\begin{array}{c}1.89 \\
(1.13-2.92)\end{array}$ & $1.70(1.13-3.03)$ & $1.48(0.95-2.2)$ & $1.41(0.99-2.34)$ & NS \\
\hline $\begin{array}{l}\text { Composición } \\
\text { Quístico (\%) } \\
\text { Mixto sólido/quístico (\%) } \\
\text { Sólido (\%) }\end{array}$ & $\begin{array}{c}100 \\
0 \\
0\end{array}$ & $\begin{array}{c}29 \\
71 \\
0\end{array}$ & $\begin{array}{c}3 \\
22 \\
75\end{array}$ & $\begin{array}{c}3 \\
28 \\
69\end{array}$ & $\begin{array}{c}1 \\
20 \\
79\end{array}$ & $<0.001^{*}$ \\
\hline $\begin{array}{l}\text { Márgenes } \\
\text { Lisos o mal definidos (\%) } \\
\text { Lobulados e irregulares (\%) } \\
\text { Extensión extratiroidea (\%) }\end{array}$ & $\begin{array}{c}100 \\
0 \\
0\end{array}$ & $\begin{array}{c}100 \\
0 \\
0\end{array}$ & $\begin{array}{c}99 \\
1 \\
0\end{array}$ & $\begin{array}{l}78 \\
16 \\
6\end{array}$ & $\begin{array}{l}35 \\
42 \\
23\end{array}$ & $<0.001^{*}$ \\
\hline Rigidez $(\mathrm{kPa})^{\ddagger}$ & $\begin{array}{c}11.86 \\
(9.41-22.40)\end{array}$ & $\begin{array}{c}15.96 \\
(9.76-21.84)\end{array}$ & $\begin{array}{c}18.48 \\
(12.87-27.81)\end{array}$ & $\begin{array}{c}18.80 \\
(13.03-30.94)\end{array}$ & $\begin{array}{c}25.52 \\
(16.53-42.60)\end{array}$ & $<0.001^{\star *}$ \\
\hline $\begin{array}{l}\text { Bethesda } \\
\text { I (\%) } \\
\text { II-III (\%) } \\
\text { IV-VI (\%) }\end{array}$ & $\begin{array}{l}20 \\
70 \\
10\end{array}$ & $\begin{array}{c}21 \\
74 \\
5\end{array}$ & $\begin{array}{l}20 \\
61 \\
19\end{array}$ & $\begin{array}{l}25 \\
51 \\
24\end{array}$ & $\begin{array}{l}20 \\
38 \\
42\end{array}$ & $\begin{aligned} & N S \\
< & 0.001^{*} \\
< & 0.001^{*}\end{aligned}$ \\
\hline
\end{tabular}

siguiente forma: $22 \%$ se clasificó como Bethesda I, $64 \%$ como Bethesda II-III y $14 \%$ como Bethesda IV-VI.

Al comparar los grupos TI-RADS 5 versus TI-RADS 1-4 y los valores de la elastografía con la clasificación por el sistema Bethesda, se observó que la rigidez del nódulo se incrementó dependiendo de la clasificación citopatológica, de tal forma que los nódulos TI-RADS 5 y categoría Bethesda baja (II-III) tuvieron una mediana de $21.1 \mathrm{kPa}(14.3$ a $33 \mathrm{kPa})$, mientras que la mediana fue de $35.9 \mathrm{kPa}(22.6$ a $68.6 \mathrm{kPa})$ en aquellos clasificados con categoría Bethesda alta (IV-VI), $p<0.001$. En cuanto a los nódulos TIRADS 1-4, aquellos con categoría Bethesda II-III tuvieron una mediana de $17.7 \mathrm{kPa}(12.1-27 \mathrm{kPa})$, mientras que aquellos estratificados como Bethesda IV-VI tuvieron una mediana de $21.6 \mathrm{kPa}$ (13.7 a $38.5 \mathrm{kPa}), \mathrm{p}=0.009$.

Mediante curva ROC (Figura 1) se obtuvo que el punto de corte para predicción de nódulos TIRADS 5 que requieren exploración quirúrgica es $32.5 \mathrm{kPa}$, con un área bajo la curva ROC (AUROC) de 0.69 (IC 95\% $=0.63$ 0.76), especificidad de $75 \%$, sensibilidad de $57 \%$, valor predictivo negativo de $60 \%$ y valor predictivo positivo de $72 \%$. Los nódulos con un valor mayor a este punto tuvieron una razón de momios de 3.97 (IC $95 \%$ = 2.76.8) para tener un resultado Bethesda IV-VI.
El punto de corte para predicción de nódulos TI-RADS 1-4 que requieren exploración quirúrgica es de $21.5 \mathrm{kPa}$, con un AUROC de 0.59 (IC $95 \%=$ 0.52-0.66), sensibilidad de $51 \%$, especificidad de $63 \%$, valor predictivo negativo de $78 \%$ y valor predictivo positivo de $31 \%$. Los nódulos con un valor mayor a este punto tuvieron una razón de momios de 2.53 (IC $95 \%=1.43-4.48$ ) de tener un resultado Bethesda IV-VI (Figura 2).

\section{Discusión}

En la evaluación de este trabajo se observó que la escala TI-RADS de la ACR para la clasificación de los nódulos tiroideos presentó alta especificidad, pero baja sensibilidad, lo cual es útil en una prueba de tamizaje; sin embargo, en nódulos tiroideos TI-RADS 5 la sensibilidad de la prueba es muy baja, lo que lleva a la realización de numerosas BAAF innecesarias. ${ }^{14}$ En este estudio, en $48.6 \%$ de las biopsias se registró un resultado benigno o inconcluso $(20.1 \%$ se clasificaron con Bethesda I y $28.5 \%$, con Bethesda II).

Debido a lo anterior, es indispensable la utilización de modalidades complementarias de imagen ecográfica como la SWE, que combinada con los criterios 


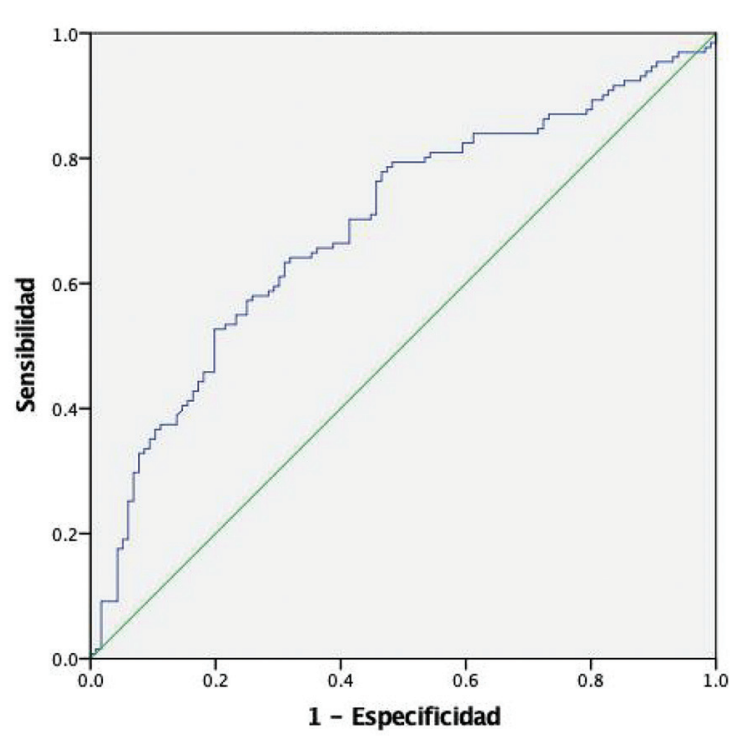

Figura 1. Curva ROC de los valores de la elastografía por ondas de corte en nódulos TI-RADS 5. El punto de corte con mayor sensibilidady especificidad para detectar nódulos que requieren cirugía fue $32.5 \mathrm{kPa}$.

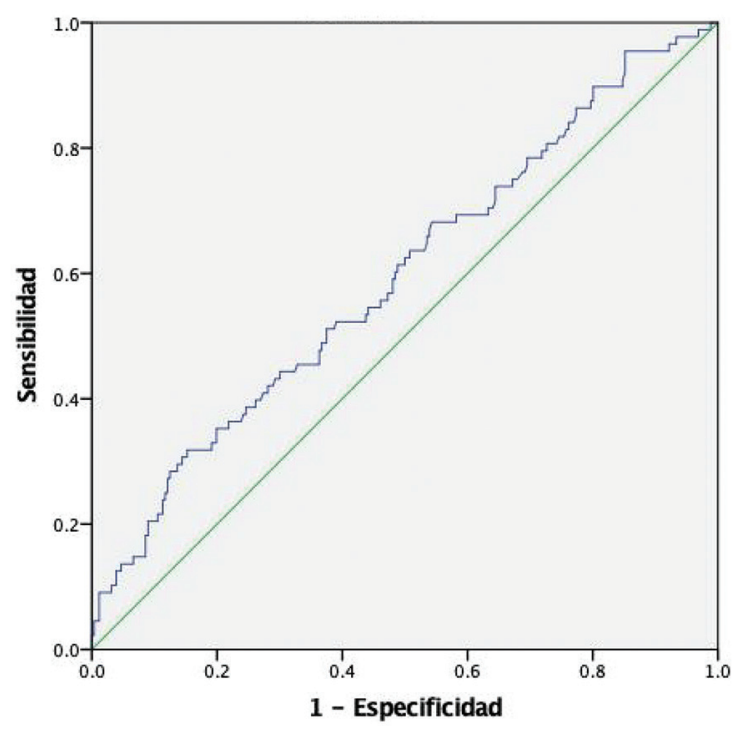

Figura 2. Curva ROC de los valores de la elastografía por ondas de corte en nódulos TI-RADS 1-4. El punto de corte con mayor sensibilidad y especificidad para detectar nódulos que requieren cirugía fue $21.5 \mathrm{kPa}$.

TI-RADS demostró una sensibilidad de $84 \%$ para la caracterización de los nódulos tiroideos.

Una de las principales características de la ecografía por escala de grises es que puede visualizarse la consistencia del nódulo; entre más sólido es un nódulo, más probabilidad de que sea maligno. Se observó que $79 \%$ de los nódulos clasificados como TI-RADS 5 fueron sólidos o casi sólidos, lo cual concuerda con los resultados de otras investigaciones en las cuales el componente sólido se relacionó con una sensibilidad de $95 \%$ y una especificidad de $48.7 \% .^{15}$

En nuestro estudio, valores superiores a $32.5 \mathrm{kPa}$ se relacionaron con mayor riesgo de resultado Bethesda IV-VI y, por lo tanto, con la necesidad de cirugía. Observamos que los valores de SWE se incrementan conforme la categoría Bethesda es mayor, por lo que la elastografía puede orientar acerca del grado de malignidad del nódulo y del lugar de obtención de la muestra para la biopsia, ya que es posible identificar el sitio con mayor rigidez y componente sólido.

Hang et al. observaron que valores menores a $22.5 \mathrm{kPa}$ predicen benignidad, lo cual concuerda con lo que encontramos en los nódulos TI-RADS 1-4 (mediana de $21.6 \mathrm{kPa}) ;{ }^{16}$ en esa investigación, el nódulo TI-RADS 5 tuvo una mediana de $31.1 \mathrm{kPa},{ }^{16}$ mientras que en el presente trabajo se determinó una mediana de $32.5 \mathrm{kPa}$. No se encontraron estudios previos que correlacionaran la clasificación ACR TI-RADS y la SWE con los mismos puntos de corte para el análisis de estas variables. En otro estudio con características similares se encontró que el punto de corte de $34.5 \mathrm{kPa}$ tuvo una sensibilidad de $76 \%$ y una especificidad de $71.1 \%$ en el diagnóstico de cáncer tiroideo y que un valor de corte de $22.30 \mathrm{kPa}$ (con sensibilidad de $82 \%$ y especificidad de $88 \%$ ) permitió diferenciar lesiones malignas de lesiones benignas. ${ }^{17}$

Una de las limitaciones de nuestro estudio consiste en que no se pudo dar seguimiento a los pacientes con un resultado Bethesda IV-VI que se sometieron a cirugía, por lo que no se dispuso del resultado definitivo del análisis histopatológico total de la pieza obtenida de la hemitiroidectomía o tiroidectomía total. Una de las fortalezas es el número de nódulos evaluados en un solo centro de referencia, lo cual disminuye la variabilidad en la evaluación ultrasonográfica e histopatológica.

Los nódulos que tuvieron un resultado Bethesda I en la biopsia fueron excluidos del análisis estadístico; representaron $21.2 \%$ del total de nódulos en los que se realizó biopsia, en contraste con 5 a $10 \%$ que se informa en otros estudios. ${ }^{6}$ Sería posible disminuir el número de biopsias no diagnósticas si se dispusiera de un resultado citológico preliminar antes de que el paciente sea dado de alta, lo que permitiría su correcta clasificación.

Derivado de los resultados de este trabajo, planteamos posteriormente analizar en esta población los factores predictores de malignidad, con la comparación del 
rendimiento de la TI-RADS sola, de la SWE sola y la combinación de las dos pruebas, ya que en otros estudios se ha demostrado que el uso conjunto puede tener una especificidad de hasta $95.8 \%$ y una sensibilidad de $76.29 \%$ en comparación con la TI-RADS sola, que tiene una especificidad de $88.24 \%$ y una sensibilidad de $89.6 \%$, o del SWE, cuya especificidad es de $83.1 \%$ y su sensibilidad de $81.4 \% .^{15}$

Una intervención que debe considerarse es la inclusión de la elastografía en los criterios TI-RADS de la ACR, y de otras asociaciones como la ATA y la KTA/ $\mathrm{KSThR},{ }^{18}$ ya que, aunque el trabajo se realizó utilizando la clasificación TI-RADS de la ACR, el uso de la elastografía per se mostró una asociación con los resultados citopatológicos.

\section{Conclusiones}

La SWE es una herramienta útil en la clasificación de los nódulos tiroideos: se observó que a mayor rigidez de estos, mayor la posibilidad de un resultado histopatológico maligno, relación que podría permitir la identificación de los pacientes que necesitarán exploración quirúrgica. Los nódulos TI-RADS 1-4 con valores de rigidez por SWE menores a $21.6 \mathrm{kPa}$ tuvieron mayor probabilidad de ser benignos, mientras que los nódulos tiroideos TI-RADS 5 con rigidez $>32.5 \mathrm{kPa}$ mostraron mayor riesgo de ser malignos.

\section{Agradecimientos}

Agradecemos a la Coordinación de Investigación en Salud del Instituto Mexicano del Seguro Social por la realización del Taller de Redacción del Artículo Científico (TRAC), durante el cual se realizó este manuscrito.

\section{Conflictos de intereses}

Los autores declaran no tener ningún conflicto de interés.

\section{Financiamiento}

Los autores no recibieron patrocinio para llevar a cabo este artículo.

\section{Responsabilidades éticas}

Protección de personas y animales. Los autores declaran que los procedimientos seguidos se conformaron a las normas éticas del comité de experimentación humana responsable y de acuerdo con la Asociación Médica Mundial y la Declaración de Helsinki.

Confidencialidad de los datos. Los autores declaran que siguieron los protocolos de su centro de trabajo sobre la publicación de datos de pacientes.

Derecho a la privacidad y consentimiento informado. Los autores obtuvieron el consentimiento informado de los pacientes o sujetos referidos en el artículo. Este documento obra en poder del autor de correspondencia.

\section{Bibliografía}

1. American Thyroid Association [sitio web]. Disponible en thyroid.org.

2. World Health Organization. Mexico. Source: Globocan 2020. [Internet]; 2019. Disponible en: http://gco.iarc.fr/today/data/factsheets/populations/484-mexico-fact-sheets.pdf

3. Wei X, Li Y, Zhang S, Gao M. Thyroid imaging reporting and data system (TI-RADS) in the diagnostic value of thyroid nodules: a systematic review. Tumour Biol. 2014:35:6769-6776.

4. Ha SM, Baek JH, Na DG, Suh CH, Chung SR, Choi YJ, et al. Diagnostic performance of practice guidelines for thyroid nodules: thyroid nodule size versus biopsy rates. Radiology. 2019;291:92-99.

5. Tessler FN, Middleton WD, Grant EG, Hoang JK, Berland LL, Teefey SA, et al. ACR Thyroid Imaging, Reporting and Data System (TI-RADS): White Paper of the ACR TI-RADS Committee. J Am Coll Radiol. 2017; 14:587-595.

6. Cibas ES, Ali SZ. The 2017 Bethesda system for reporting thyroid cytopathology. Thyroid. 2017;27:1341-1346.

7. Hambleton C, Kandil E. Appropriate and accurate diagnosis of thyroid nodules: a review of thyroid fine-needle aspiration. Int J Clin Exp Med. 2013;6:413-422.

8. Kizilkaya MC, Erozgen F, Akinci M, Kaplan R, Tuzun S, Citlak G. The predictive value of elastography in thyroid nodules and its comparison with fine-needle aspiration biopsy results. Ulus Cerrahi Derg. 2014; 30:147-152.

9. McQueen AS, Bhatia KS. Thyroid nodule ultrasound: technical advances and future horizons. Insights Imaging. 2015;6:173-188.

10. Zhao CK, Xu HX: Ultrasound elastography of the thyroid: principles and current status. Ultrasonography. 2019;38:106-124.

11. Bhatia KS, Rasalkar DP, Lee YP, Wong KT, King AD, Yuen HY, et al. Cystic change in thyroid nodules: a confounding factor for real-time qualitative thyroid ultrasound elastography. Clin Radiol. 2011;66: 799-807.

12. Veyrieres JB, Albarel F, Lombard JV, Berbis J, Sebag F, Oliver C, et al. A threshold value in shear wave elastography to rule out malignant thyroid nodules: a reality? Eur J Radiol. 2012;81:3965-3972.

13. Akobeng AK. Understanding diagnostic tests 3 : Receiver operating characteristic curves. Acta Paediatr. 2007;96:644-647.

14. Hoang JK, Middleton WD, Farjat AE, Langer JE, Reading CC, Teefey SA, et al. Reduction in thyroid nodule biopsies and improved accuracy with American College of Radiology thyroid imaging reporting and data system. Radiology. 2018;287:185-193.

15. Cantisani V, David E, Grazhdani H, Rubini A, Radzina M, Dietrich CF, et al. Prospective evaluation of semiquantitative strain ratio and quantitative 2D ultrasound shear wave elastography (SWE) in association with TIRADS classification for thyroid nodule characterization. Ultraschall Med. 2019;40:495-503.

16. Hang J, Li F, Qiao XH, Ye XH, Li A, Du LF. Combination of maximum shear wave elasticity modulus and TIRADS improves the diagnostic specificity in characterizing thyroid nodules: a retrospective study. Int J Endocrinol. 2018;2018:4923050.

17. Liu Z, Jing $H$, Han X, Shao $H$, Sun $Y X$, Wang $Q C$, et al. Shear wave elastography combined with the thyroid imaging reporting and data system for malignancy risk stratification in thyroid nodules. Oncotarget. 2017:8:43406-43416.

18. Middleton WD, Teefey SA, Reading CC, Langer JE, Beland MD, Szabunio MM, et al. Comparison of performance characteristics of American College of Radiology TI-RADS, Korean Society of Thyroid Radiology TIRADS, and American Thyroid Association Guidelines. AJR Am J Roentgenol. 2018;210:1148-1154. 